\title{
Clinical Evaluation of Halitosis Patients in Korea
}

\author{
Min-Jae Kim, Soo-Kyung Kang, Yang-Hyun Chun, Jung-Pyo Hong, Q-Schick Auh
}

Department of Orofacial Pain and Oral Medicine, School of Dentistry, Kyung Hee University, Seoul, Korea

Received April 4, 2014 Revised April 23, 2014 Accepted May 9, 2014
Correspondence to:

Q-Schick Auh

Department of Orofacial Pain and Oral

Medicine, School of Dentisty, Kyung

Hee University, 26, Kyungheedae-ro,

Dongdaemun-gu, Seoul 130-701,

Korea

Tel: +82-2-958-9418

Fax: +82-2-962-2043

E-mail: dental21@khu.ac.kr
Purpose: This study was designed to evaluate the sex and age differences in the Korean oral malodor patients.

Methods: Subjects were the patients with oral malodor (male, 2,239; female, 3,496) visited the Department of Oral Medicine, Kyung Hee University Dental Hospital from January 2007 to December 2012 were recruited to this study and measured the volatile sulfur compounds (VSCs) in the oral cavity with Halimeter.

Results: There were slightly more female than male (female : male $=3: 2$ ), and the mean values of Halimeter result were $65.79 \mathrm{ppb}$ (female) and $79.94 \mathrm{ppb}$ (male), which was more higher in male. Total patients showed normal age distribution in both gender, and the peak was the age of 26-35 in female, the age of 36-45 in male. The percentages of pseudo-halitosis were 74.7\% in female and 69.3\% in male, which was more higher in female.

Conclusions: Therefore, it was revealed that women are more likely to have oral malodor than men, and there was a difference in the largest age group between men and women. Also women had more pseudo-halitosis patient than men.

Key Words: Halimeter; Pseudo-halitosis; Volatile sulfur compounds

\section{INTRODUCTION}

Halitosis is the common term used to define an unpleasant or an offensive odor in exhaled breath, regardless of whether it originates from oral or non-oral sources. ${ }^{1)}$ Althernative terms of halitosis include bad breath, foul breath, breath odor, foul smells, feotor ex ore, breath malodor, oral malodor or offensive breath. Halitosis has been a very disturbing problem throughout all over the world beyond history, culture, race and sex. The record about status and treatment of halitosis could be found in not only the book of Hippocrates, but also ancient greek and roman documents. Halitosis is caused by a variety of reasons including but not limited to periodontal disease, bacterial coating of tongue, systemic disorders and different types of food. ${ }^{2)}$ In addition, halitosis can be detected when right after the wake up in the morning, empty stomach, during a period or after ingesting specific food or drug which released from lungs. This is called physiologic halitosis which must be classified with pathologic halitosis. ${ }^{3-6)}$

Halitosis is a widespread problem in the general population throughout the world. ${ }^{7)}$ It has been estimated that 90 million Americans or approximately 30\% of the US population currently suffers from bad breath on a regular basis. ${ }^{8,9)}$ In other studies, $20 \%-60 \%$ of the population suffers from chronic oral malodor, and in approximately half of these individuals, the problem becomes serious enough to create personal discomfort and social embarrassment. ${ }^{8,10,11)}$ Based on different methodologies, the prevalence of oral malodor is unclear. There is a considerable amount of research regarding halitosis. In the Eastern world, however, there is a relative lack of good clinical data describing the aetiology and characteristics of bad breath for large groups of patients.

The aim of this study was to analyze the characteristics of halitosis in Korea of a large group of patients with a volatile sulfur compounds (VSCs) measuring device (Halimeter; Interscan Corp., Chatsworth, CA, USA). 


\section{MATERIALS AND METHODS}

\section{Subject Selection}

This study utilized data recorded in the Department of Oral Medicine, Kyung Hee University Dental Hospital From January 2007 to December 2012, a total of 5,735 patients who complained of halitosis visited the clinic. This retrospective study was approved by Kyung Hee University Dental Hospital Institutional Review Board (No. KMC IRB 1310-2). The subjects of $60.9 \%$ were female with the average age was 41.9 years and 39.1\% were male with the average age was 42.7 years. Informed consent was not deemed necessary because of the retrospective nature of this study. All the patients were assessed through an identical manner. Patients were asked not to eat garlic, onions or spicy food before their appointment. They were also instructed to refrain from drinking alcohol or coffee, and from smoking during the 12-hour period before their appointment. On the morning of the appointment, patients were asked not to use chewing gum, mints, drops, scents or mouth rinses. Toothbrushing with water and breakfast were allowed in order to avoid confusion between breath malodour and morning bad breath. All measurements were recorded at least 2 hours after eating or drinking or oral hygiene. The complete examination of each patient did not take longer than 30 minutes.

\section{Clinical Examination}

The oral cavity was examined, paying attention to caries, plaque accumulation, gingival inflammation, periodontal pockets, removable appliances and tongue coating. The patients who had caries, calculus deposition or periodontal diseases were all excluded in this study and only tongue coated patients were included.

\section{Halimeter Measurements}

Mouth air was examined with a VSCs measuring device (model RH-17R, Halimeter) (Fig. 1). Measurements were carried out three times $(1 \mathrm{~cm}, 2 \mathrm{~cm}, 3 \mathrm{~cm})$ by inserting a disposable tube into the subject's mouth, above the posterior part of the dorsum of the tongue without touching the oral mucosa or the tongue. The subject kept the mouth sealed for three minutes and was allowed to breathe with nose during sampling. The three time results were averaged.

\section{Statistical Analysis}

Statistical analysis was carried out using SPSS 10.0E software (SPSS Inc., Chicago, IL, USA). Independent sample t-test and Mann-Whitney U test were used to detect differences in the mean VSCs values between male and female according to age group. Differences between male and female with Halimeter values of over $75 \mathrm{ppb}$ and $150 \mathrm{ppb}$ were examined using chi-square test. The data were analyzed for means and frequency distributions. A p-value $<0.05$ was taken as significant.

\section{RESULTS}

\section{Descriptive Information}

Thus far, the data of the 5,735 consecutive patients who visited the Department of Oral Medicine, Kyung Hee University Dental Hospital have been analysed. There were slightly more females $(n=3,496,60.9 \%)$ than males $(n=2,239$, $39.1 \%$ ). The patient's age was ranged from 2 to 97 years. In Table 1 , we can notice almost $4.6 \%$ of the patients were under the age of 15 which is consistent with the result of Quirynen et al. ${ }^{12)}$ As you can see in Fig. 2, total patients showed normal age distribution in both gender, and the largest percentage were the age of 26-35 years in female and the age of $36-45$ years in male.

\section{Halitosis Measurements}

Most patients showed the VSCs level of under $75 \mathrm{ppb}$ in both genders. The VSCs level was slightly higher for male (mean, 79.94 ppb; standard deviation [SD], 107.09) than for female (mean, 65.79 ppb; SD, 89.48) (Table 2).

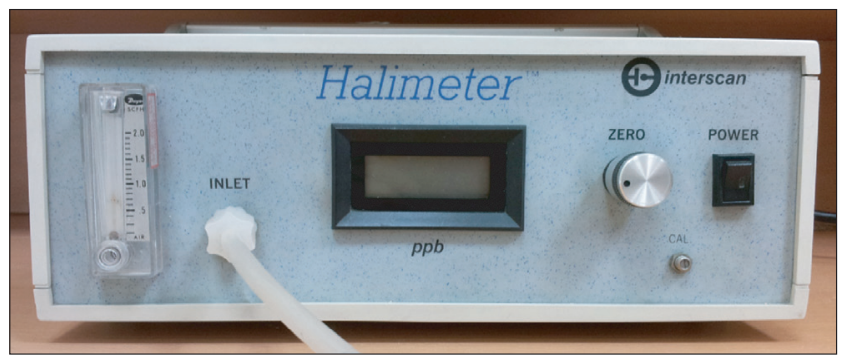

Fig. 1. Halimeter (Interscan Corp.). 
Table 1. The subject's distribution by gender and age

\begin{tabular}{lccc}
\hline Age $(y)$ & Female & Male & Total \\
\hline$\leq 15$ & 156 & 107 & $263(4.6)$ \\
$16-25$ & 485 & 303 & $788(13.7)$ \\
$26-35$ & 806 & 410 & $1,216(21.2)$ \\
$36-45$ & 696 & 504 & $1,200(20.9)$ \\
$46-55$ & 521 & 399 & $920(16.0)$ \\
$56-65$ & 406 & 251 & $657(11.5)$ \\
$66-75$ & 284 & 146 & $430(7.5)$ \\
$\geq 76$ & 142 & 119 & $261(4.6)$ \\
Total & 3,496 & 2,239 & $5,735(100)$ \\
\hline
\end{tabular}

Values are presented as number or number (\%).

Table 2. Volatile sulphur compounds levels (Halimeter) for male and female patients

\begin{tabular}{|c|c|c|c|}
\hline \multirow{2}{*}{$\begin{array}{l}\text { Halimeter value } \\
\qquad(p p b)\end{array}$} & Female & Male & \multirow{2}{*}{ Total } \\
\hline & $65.79 \pm 89.48$ & $79.94 \pm 107.09$ & \\
\hline $0-75$ & 2,613 & 1,551 & $4,164(72.6)$ \\
\hline $76-150$ & 535 & 392 & 927 (16.2) \\
\hline $151-225$ & 196 & 140 & $336(5.9)$ \\
\hline $226-300$ & 71 & 61 & $132(2.3)$ \\
\hline $301-375$ & 44 & 37 & 81 (1.4) \\
\hline $376-450$ & 9 & 22 & $31(0.5)$ \\
\hline$>450$ & 28 & 36 & 64 (1.1) \\
\hline Total & 3,496 & 2,239 & $5,735(100)$ \\
\hline
\end{tabular}

Values are presented as mean \pm standard deviation, number, or number (\%).

Table 3. Results of patients' classification

\begin{tabular}{crr}
\hline Class & \multicolumn{1}{c}{ Female } & \multicolumn{1}{c}{ Male } \\
\hline I (genuine halitosis) & $883(25.3)$ & $688(30.7)$ \\
II (pseudo-halitosis) & $2,613(74.7)$ & $1,551(69.3)$ \\
\hline
\end{tabular}

Values are presented as number (\%).

Fig. 3 shows this result in a bar graph. The subject whose VSCs level of under $75 \mathrm{ppb}$, which means not an objective halitosis, accounts for about 70\% male and female both. In objective halitosis, which means over 150 ppb VSCs level male patients were slightly more than female patients.

In Table 3, the percentages of classes I (genuine halitosis) and II (pseudo-halitosis) halitosis were 25.3\% and 74.7\% in female, and 30.7\% and 69.3\% in male, respectively. Therefore, the most prevalent class of halitosis in the patients were pseudo-halitosis in both genders.

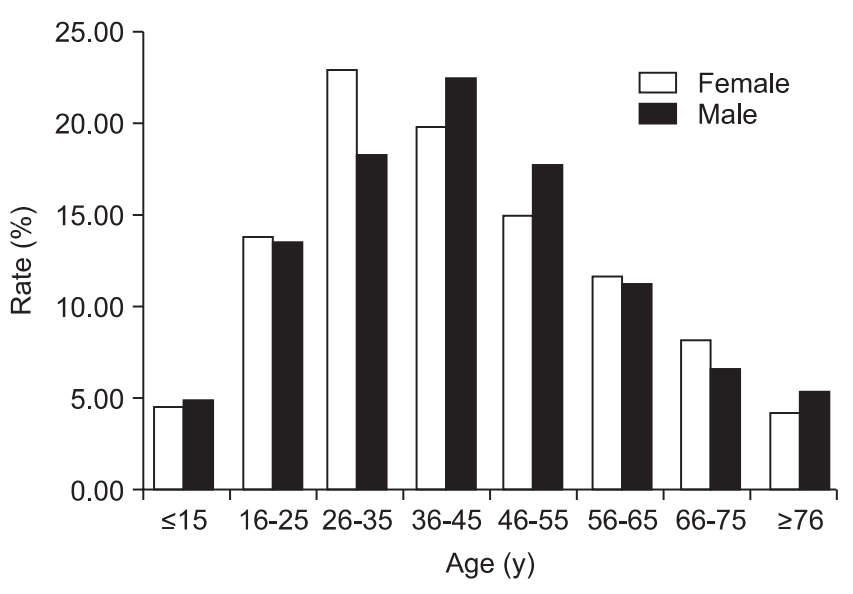

Fig. 2. General characteristics: age of patients.

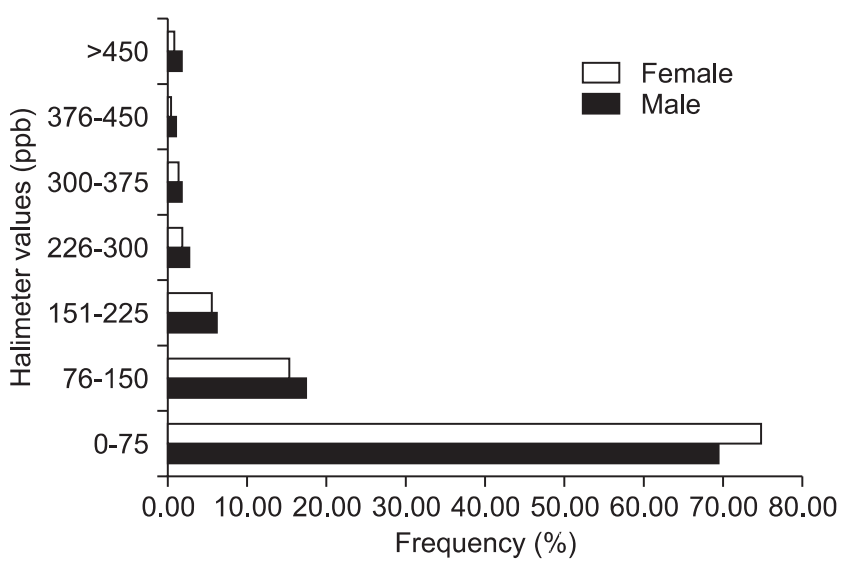

Fig. 3. Volatile sulphur compounds levels (Halimeter) for male and female patients.

\section{Correlations}

A significant correlation was found between average VSCs values and each gender of age group (Table 4). Significant differences were found for the mean VSCs values between female and male the age group of 26-35 years, 36-45 years, 46-55 years $(p<0.01)$, and $66-75$ years $(p<0.05)$ but not between the age group of $\leq 15$ years, $16-25$ years, $56-65$ years, and $\geq 76$ years.

The result of statistical analysis which the subjects were devided according to two standards ( $>75 \mathrm{ppb},>150 \mathrm{ppb}$ ) is in Table 5. The proportion of patients who had over 75 ppb VSCs level were 894 (25.6\%) in female, 692 (30.9\%) in male, which had significant statistical differences $(\mathrm{p}<0.01)$. And the proportion of patients who had over $150 \mathrm{ppb}$ VSCs level were $349(10.0 \%)$ in female, 298 (13.3\%) in male, which had also significant statistical differences $(\mathrm{p}<0.01)$. 
Table 4. VSCs distribution by age group based on gender

\begin{tabular}{|c|c|c|c|c|c|c|c|c|}
\hline & \multicolumn{8}{|c|}{ Age (y) } \\
\hline & $\leq 15$ & $16-25$ & $26-35$ & $36-45$ & $46-55$ & $56-65$ & $66-75$ & $\geq 76$ \\
\hline Number & 263 & 788 & 1,216 & 1,200 & 920 & 657 & 430 & 261 \\
\hline Female & $90.38 \pm 105.76$ & $72.24 \pm 104.12$ & $66.99 \pm 86.26 * \star$ & $66.05 \pm 82.14^{\star \star}$ & $61.11 \pm 76.04^{* *}$ & $71.99 \pm 117.95$ & $54.06 \pm 63.01 *$ & $48.96 \pm 49.29$ \\
\hline Male & $92.55 \pm 119.53$ & $75.6 \pm 96.97$ & $77.58 \pm 106.52^{\star *}$ & $84.48 \pm 119.24 * *$ & $80.14 \pm 104.96^{* *}$ & $83.55 \pm 114.35$ & $71.06 \pm 68.86^{*}$ & $71.68 \pm 97.81$ \\
\hline
\end{tabular}

VSCs, volatile sulfur compounds.

Values are presented as number or mean \pm standard deviation.

Significant differences between paired items within each age group: ${ }^{*} p<0.05 .{ }^{* *} p<0.01$.

Table 5. Chi-square test between VSCs (>75 group, >150 group) and gender

\begin{tabular}{lll}
\hline & $>75 \mathrm{ppb}$ & $>150 \mathrm{ppb}$ \\
\hline Female & $894(25.6)^{\star \star}$ & $349(10.0)^{\star \star}$ \\
Male & $692(30.9)^{\star \star}$ & $298(13.3)^{\star \star}$ \\
\hline
\end{tabular}

Values are presented as number (\%).

${ }^{* *} p<0.01$.

\section{DISCUSSION}

Halitosis is a recognizable condition that warrants professional attention by dental care providers. Growing commercial interest as evidenced by the variety of products advertised for managing "bad breath" reflects the significant concern of a large percentage of the general population about this condition. ${ }^{13,14)}$

Halitosis occurs when the human, bacteria and substrate are interacted together. It is known that in the human exhaled air contains over 400 kinds of VSCs, so the concentration and distribution of this volatile compounds affect the tendency and intensity of halitosis. So it is important to evaluate objectively various volatile compounds affecting halitosis to diagnosis the oral malodor. In this study, an Halimeter device was used to measure VSCs. It is a portable gas chromatograph invented for measuring halitosis through VSCs measurement. It does not need specialized staff and does not take much time to measure. It is not heavy and does not take much space; therefore, it is a useful tool for clinical and research usage.

The manufacturer of Halimeter had not stated a definite value of ppb for normal reading for many years. Yaegaki and Sanada ${ }^{15)}$ recommended $75 \mathrm{ppb}$ as a perceived level of malodor in mouth air. Miyazaki et al. ${ }^{16)}$ also utilized the same standard in their survey of the general population for halitosis in Japanese. Recently, the manufacturer suggested $150 \mathrm{ppb}$ or below as a normal reading in their instructions (http://www.halimeter.com). To make this study more objective, we included both evaluation methods in our study.

To the best of our knowledge, the present study is the first time that the aetiology and characteristics of halitosis have been studied in Korea, and for a group of patients as large as this one. In the present study, we found a $72.6 \%$ prevalence of self-perceived oral malodor which is higher than usually reported, ${ }^{17-19)}$ and may result from the difference of measurement methods ranging from self-reported oral malodor to objective measurements such as VSCs monitoring or organoleptic scores and the sampling design. The patients who visited the clinic with chief complaint of oral malodor were slightly more females than males (female : male $=3: 2$ ), which is consistent with the result of Park et al. ${ }^{20)}$ The average values of Halimeter result were 65.79 ppb (female) and $79.94 \mathrm{ppb}$ (male) which was more higher in male. The reason of this result is because the proportion of under $75 \mathrm{ppb}$ VSCs level is relatively higher in female than male, on the contrary, the proportion of over 150 ppb VSCs level is relatively higher in male than female. Also the slightly higher VSCs level of male than female in all age group could cause this result. Halitosis has a complex nature and is a sociopsychosomatic disease. The diversity of the results between our study and other reports may be related to cultural, religious, economic, psychologic and lifestyle differences. To find the reasons for the diversities, all factors must be studied comparatively in a distinct multicentre study.

The term pseudo-halitosis is used when no breath malodour can be perceived, and yet the patient is convinced that he suffers from it. If after a diagnosis of pseudo-halitosis the patient still believes that there is bad breath, one can speak about halitophobia. ${ }^{21)}$ 
Even when our 5,735 patients do not represent the general population because they have actually looked for help for their halitosis problem, it must be noticed that the proportion of patients with pseudo-halitosis or halitophobia has grown over the years. In 2005, Vandekerckhove et al. ${ }^{22)}$ showed that the pseudo-halitosis/halitophobia rate was 7.6\% and it reached at $15.7 \%$ in $2009 .{ }^{12)}$

One possible reason for the increase in the percentage of patients with imaginary halitosis is the high amount of advertising regarding fresh breath, which is now available through the media, especially the Internet. It is not infrequent that patients notice a bad taste in their mouth, and wrongly assume they have breath malodour. Bad taste can be further strengthened when tonsil stones (tonsilloliths), which smell very bad, but do not necessarily give rise to halitosis, are coughed up. ${ }^{23)}$

Consistent with previous findings, about 2/3 of the halitophobics were women. ${ }^{12,24)}$ The higher proportion of female halitophbics helps to partly explain the significantly higher Halimeter values observed for men, although some previous studies have also mentioned some kind of relationship between gender and halitosis levels. ${ }^{25,26)}$

The largest proportion of patient's age group was 26-35 in female and 36-45 years in male, this was not consistent with previous studies, ${ }^{13,16,20,27,28)}$ the number of oral malodor patients was significantly increasing with age, which may show an association between increased VSCs level with age. ${ }^{16)}$ The reason of this tendency is probably because in the modern society, the more population have a social activities, the more interest about halitosis which could interfere smooth human relationship have been grown in young generation. But typically true halitosis increases with aging because the risk of periodontal disease or xerostomia is elevated together. So it is suggested that consideration and management of chronic diseases should be done prior to treat halitosis in older generation.

We found that male and female were significantly different in the prevalence of oral malodor in each age groups. This finding is similar to the studies of university students in Brazil, ${ }^{27)}$ Poland ${ }^{29)}$ and dental students in Saudi-Arabia, ${ }^{30)}$ but different with the studies in Kuwait ${ }^{13)}$ and Turkey ${ }^{31)}$ patients, which showed that males suffered from oral malodor more often than females. Overall male VSCs level was higher tendency than female VSCs level, especially the age group of 26-35 years, 36-45 years, 46-55 years, and 66-75 years showed statistical differences between male and female. Also the proportion of halitosis patient on the basis of over $75 \mathrm{ppb}$ and over $150 \mathrm{ppb}$ VSCs level showed statistical differences between male and female. The reason of male had more proportion of true halitosis patient than female is, because men have higher rate of smoking and alcohol ingestion than women. Smoking and alcohol ingestion may result in transient oral malodor because some substances can cause xerostomia and alcoholic beverages are known to produce volatile compounds, acetaldehyde and other odorous byproducts by oxidation of alcohol in the mouth and liver. ${ }^{32,33)}$

In the present study, despite the limitation of without consideration of malodor risk factors (changes in dietary habits, decreased salivary flow, change in the quality of saliva, an inability to physically cope with oral hygiene techniques and overnight denture wear) and result from a different practitioner because of long term data collection period during 2007 to 2012, we can understand the tendency of halitosis and its association with age and sex through a large population in Korea. It is important to enhance the knowledge of oral and general health among common people through this result, to seek collaboration between oral health care professionals including dentists, Department of Otolaryngology specialists, gastroenterologists, nutritionists and psychologists, which will enable an appropriate multidisciplinary approach for management of this condition.

This study was designed to evaluate the sex and age differences in the Korean oral malodor patients. Subjects were the patients with oral malodor (male, 2,239; female, 3,496) visited the Department of Oral Medicine, Kyung Hee University Dental Hospital from January 2007 to December 2012 were recruited to this study and measured the VSCs in the oral cavity with Halimeter.

After the examination, the results were catagorized and analyzed statistically.

The results were as follows:

1. There were slightly more female than male (female : male= $3: 2$ ), and the mean values of Halimeter result were 65.79 ppb (female) and $79.94 \mathrm{ppb}$ (male), which was more higher in male. 
2. Total patients showed normal age distribution in both gender, and the peak was the age of 26-35 years in female, the age of $36-45$ years in male.

3. The percentages of pseudo-halitosis were $74.7 \%$ in female and 69.3\% in male, which was more higher in female.

Therefore, it was revealed that women are more likely to have oral malodor than men, and there was a difference in the largest age group between men and women. Also women had more pseudo-halitosis patient than men. Further investigations with same practitioner to reduce the measuring error and considerations about the factors influence on the oral malodor have to be followed.

\section{CONFLICT OF INTEREST}

No potential conflict of interest relevant to this article was reported.

\section{REFERENCES}

1. Bornstein MM, Kislig K, Hoti BB, Seemann R, Lussi A. Prevalence of halitosis in the population of the city of Bern, Switzerland: a study comparing self-reported and clinical data. Eur J Oral Sci 2009;117:261-267.

2. Rösing CK, Loesche W. Halitosis: an overview of epidemiology, etiology and clinical management. Braz Oral Res 2011;25:466471.

3. Tonzetich J. Production and origin of oral malodor: a review of mechanisms and methods of analysis. J Periodontol 1977;48:13-20.

4. Rosenberg M. Bad breath, diagnosis and treatment. Univ Tor Dent J 1990;3:7-11.

5. Tonzetich J. Oral malodour: an indicator of health status and oral cleanliness. Int Dent J 1978;28:309-319.

6. van Steenberghe D. Breath malodor. Curr Opin Periodontol 1997;4:137-143.

7. Youngnak-Piboonratanakit P, Vachirarojpisan T. Prevalence of self-perceived oral malodor in a group of thai dental patients. $\mathrm{J}$ Dent (Tehran) 2010;7:196-204.

8. Rayman S, Almas K. Halitosis among racially diverse populations: an update. Int J Dent Hyg 2008;6:2-7.

9. Cortelli JR, Barbosa MD, Westphal MA. Halitosis: a review of associated factors and therapeutic approach. Braz Oral Res 2008;22 (Suppl 1):44-54.

10. Bosy A. Oral malodor: philosophical and practical aspects. J Can Dent Assoc 1997;63:196-201.

11. Brunette DM, Proskin HM, Nelson BJ. The effects of dentifrice systems on oral malodor. J Clin Dent 1998;9:76-82.

12. Quirynen M, Dadamio J, Van den Velde S, et al. Characteristics of 2000 patients who visited a halitosis clinic. J Clin Periodontol 2009;36:970-975.
13. Al-Ansari JM, Boodai H, Al-Sumait N, Al-Khabbaz AK, AlShammari KF, Salako N. Factors associated with self-reported halitosis in Kuwaiti patients. J Dent 2006;34:444-449.

14. Tessier JF, Kulkarni GV. Bad breath: etiology, diagnosis and treatment. Oral Health 1991;81:19-22, 24.

15. Yaegaki K, Sanada K. Effects of a two-phase oil-water mouthwash on halitosis. Clin Prev Dent 1992;14:5-9.

16. Miyazaki H, Sakao S, Katoh Y, Takehara T. Correlation between volatile sulphur compounds and certain oral health measurements in the general population. J Periodontol 1995;66:679-684.

17. Loesche WJ, Kazor C. Microbiology and treatment of halitosis. Periodontol 2000 2002;28:256-279.

18. ADA Council on Scientific Affairs. Oral malodor. J Am Dent Assoc 2003;134:209-214

19. Iwanicka-Grzegorek E, Michalik J, Kepa J, Wierzbicka M, Aleksinski M, Pierzynowska E. Subjective patients' opinion and evaluation of halitosis using halimeter and organoleptic scores. Oral Dis 2005;11(Suppl 1):86-88.

20. Park MS, Kim YG, Jung SC, Lee SW. Epidemiologic study on oral malodor for Korean. Korean J Oral Med 2001;26:107-114.

21. Yaegaki K, Coil JM. Examination, classification, and treatment of halitosis; clinical perspectives. J Can Dent Assoc 2000;66:257-261.

22. Vandekerckhove B, Quirynen M, van Steenberghe D. An inventory study on a randomized group of 1000 patients visiting a multidisciplinary breath odor clinic at a university hospital. Oral Dis 2005;11(Suppl 1):98-99.

23. Pruet CW, Duplan DA. Tonsil concretions and tonsilloliths. Otolaryngol Clin North Am 1987;20:305-309.

24. Seemann R, Bizhang M, Djamchidi C, Kage A, Nachnani S. The proportion of pseudo-halitosis patients in a multidisciplinary breath malodour consultation. Int Dent J 2006;56:77-81.

25. Morita M, Wang HL. Relationship between sulcular sulfide level and oral malodor in subjects with periodontal disease. J Periodontol 2001;72:79-84.

26. Rosenberg M, Leib E. Experiences of an Israeli malodor clinic. In: Rosenberg M, ed. Bad breath: research perspectives. Tel-Aviv: Ramot Publishing; 1995. pp. 137-148.

27. Nadanovsky P, Carvalho LB, Ponce de Leon A. Oral malodour and its association with age and sex in a general population in Brazil. Oral Dis 2007;13:105-109.

28. Settineri S, Mento C, Gugliotta SC, et al. Self-reported halitosis and emotional state: impact on oral conditions and treatments. Health Qual Life Outcomes 2010;8:34.

29. Paradowska A, Marczewski B, Pawlowska-Cierniak E. Self-perception of halitosis among students of Wroclaw Medical University. Adv Clin Exp Med 2007;16:543-548.

30. Almas $\mathrm{K}, \mathrm{Al}$-Hawish $\mathrm{A}, \mathrm{Al}-\mathrm{Khamis} \mathrm{W}$. Oral hygiene practices, smoking habit, and self-perceived oral malodor among dental students. J Contemp Dent Pract 2003;4:77-90.

31. Nalcaci R, Baran I. Factors associated with self-reported halitosis (SRH) and perceived taste disturbance (PTD) in elderly. Arch Gerontol Geriatr 2008;46:307-316.

32. Hughes FJ, McNab R. Oral malodour--a review. Arch Oral Biol 2008;53(Suppl 1):S1-S7.

33. Dal Rio AC, Nicola EM, Teixeira AR. Halitosis--an assessment protocol proposal. Braz J Otorhinolaryngol 2007;73:835-842. 